\title{
ON THE EXISTENCE OF RADIAL LIMITS
}

\author{
Martha Guzmán-Partida — Carlos Robles-Corbala
}

\begin{abstract}
We discuss conditions that ensure the existence of radial limits a.e. for harmonic functions defined on the unit disc $D$. We give an example of a Banach-valued harmonic function without radial limits at almost every point on the boundary of $D$.
\end{abstract}

\section{Introduction}

A holomorphic function in the unit disc $D$ can behave very badly near the boundary $\partial D$, in general,. A simple illustration of this fact can be seen by considering the following example (see [6]).

Let $f: D \rightarrow \mathbb{C}$, be defined by $f(z)=\sum_{n=0}^{\infty} z^{n !}$. If we consider points $\omega_{\alpha}=e^{2 \pi i \alpha}$, where $\alpha=p / q$ with $p, q \in \mathbb{N}$ and $(p, q)=1$, we see that for $z=r \omega_{\alpha}$, $0<r<1$, we can write

$$
f(z)=\sum_{n=1}^{q-1} z^{n !}+\sum_{n=q}^{\infty} r^{n !}
$$

Taking $M=2 q+N$, where $N$ is an arbitrary positive integer, we obtain that

$$
|f(z)|>\sum_{n=q}^{M} r^{n !}-\sum_{n=1}^{q-1}|z|^{n !}>(M-q) r^{M !}-(q-1) \rightarrow M-2 q+1=N+1
$$

as $r \rightarrow 1^{-}$. This implies that $f$ does not have radial limits in the set $W$ of points $\omega_{\alpha}$, which is a denumerable and dense subset of $\partial D$. With much more effort, it can be proved that $f$ does not have radial limit in any point of $\partial D$ (a beautiful proof of this fact can be seen in [1, Th. 11]).

In this context, a natural question emerges: if $f$ is a holomorphic function in $D$, what are the conditions on $f$ that guarantee that $f$ will converge, in an appropriate sense, to boundary values $f\left(e^{i \theta}\right)$ on $\partial D$ ? An answer to this question is given by Fatou's theorem, a remarkable result in the theory of functions of one-complex variable originally proved in [5]. This theorem states that a bounded

(C) 2014 Mathematical Institute, Slovak Academy of Sciences.

2010 Mathematics Subject Classification: 31A05, 31A20.

Keywords: harmonic functions, Fatou's theorem. 
holomorphic function $f$ defined on $D$ has radial limits almost everywhere on $\partial D$. There is a generalization of this result for harmonic functions: every bounded harmonic function in $D$ has radial limits a.e. on the boundary of $D$ - it is also known as Fatou's theorem. Even more, the study of the behaviour on the boundary of $D$ can be extended not only in radial direction but also in the non-tangential sense, which means, to approach the boundary by means of the so-called Stoltz regions that, roughly speaking, are regions inside the disc with peaks in the boundary point. Fatou's theorem can also be proved in this context, and it is a classical result in the theory of harmonic and analytic functions. A. Zy g mund in 8] made a non-trivial construction of an analytic and bounded function $F$ on the unit disc such that for almost every $\theta \in[0,2 \pi), F(z)$ does not have a limit when $z$ moves along a curve $\gamma_{\theta}$ which is tangent to the unit circle at $e^{i \theta}$.

We can also consider harmonic functions defined on the unit disc with values in a Banach space $X$. The question now is if we might assure the existence of radial limits for this kind of functions. In the next section, we present an example that answers, in a negative sense, our question.

\section{Main result}

Let $X$ be a Banach space. We say that a function $\varphi: D \rightarrow X$ is harmonic if $\varphi$ has continuous partial derivatives of order 2 (in the sense of the norm of $X$ ) on $D$ and satisfies the Laplace's equation, that is, $\Delta \varphi=0$.

For $r>0$ and $t \in[0,1]$, we will denote by $P_{r}(t)$ the Poisson kernel in the unit disc, namely

$$
P_{r}(t)=\frac{1-r^{2}}{1+r^{2}-2 r \cos 2 \pi t} .
$$

Since harmonicity in the sense defined above is equivalent to weak harmonicity, i.e., the scalar functions $\left\langle\varphi, x^{*}\right\rangle$ are harmonic in the classical sense for each $x^{*} \in X^{*}$, it is very simple to show that if a function $f:[0,1] \rightarrow X$ is Bochner integrable with respect to the Lebesgue measure, then

$$
P(f)\left(r e^{2 \pi i t}\right)=\int_{0}^{1} P_{r}(t-s) f(s) d s
$$

is an $X$-valued harmonic function on $D$. In fact, if $T: L^{1}[0,1] \rightarrow X$ is a linear and continuous operator on the scalar space of Lebesgue integrable functions on $[0,1]$, then the function

$$
\Psi\left(r e^{2 \pi i t}\right):=T\left(P_{r}(t-\cdot)\right)
$$

is also an $X$-valued harmonic function on $D$. 


\section{ON THE EXISTENCE OF RADIAL LIMITS}

When $g:[0,1] \rightarrow \mathbb{C}$ is a Lebesgue integrable function, it is well-known that $\lim _{r \rightarrow 1} P(g)\left(r e^{2 \pi i t}\right)=g\left(e^{2 \pi i t}\right)$ a.e. for $t \in[0,1]$ (see for example, [3]). This and the previous observations will allow us to show the following result.

THEOREM 1. There exists a Banach space $X$ and a harmonic and bounded function $\varphi: D \rightarrow X$ such that $\varphi$ does not have radial limits almost everywhere on $\partial D$.

Proof. Let $X=c_{0}$, the space of complex sequences be converging to 0 . Consider the norm $\|\cdot\|_{\infty}$ in $c_{0}$, that is, for a sequence $x=\left(x_{n}\right)_{n=0}^{\infty},\|x\|_{\infty}=\sup _{n \geq 0}\left|x_{n}\right|$.

Let $T: L^{1}[0,1] \rightarrow c_{0}$ be the linear and continuous operator defined by

$$
T(f)=\left(\int_{[0,1]} f(\theta) \sin \left(2^{n} \pi \theta\right) d \theta\right)_{n=0}^{\infty} .
$$

Now, let $\varphi$ be the Poisson integral of $T$, that is $\varphi: D \rightarrow c_{0}$ such that

$$
\varphi\left(r e^{2 \pi i t}\right)=T\left(P_{r}(t-\cdot)\right) .
$$

Then, $\varphi$ is a harmonic and bounded function on $D$.

We will show that there exists a Lebesgue measurable subset $E$ of $[0,1]$ with positive Lebesgue measure such that $\lim _{r \rightarrow 1} \varphi\left(r e^{2 \pi i t}\right)$ does not exist for every $t \in E$ with respect to the norm $\|\cdot\|_{\infty}$.

Notice that

$$
\varphi\left(r e^{2 \pi i t}\right)=\left(\int_{[0,1]} P_{r}(t-\theta) \sin \left(2^{n} \pi \theta\right) d \theta\right)_{n=0}^{\infty} .
$$

Now, for every $n=0,1,2, \ldots$,

$$
\int_{[0,1]} P_{r}(t-\theta) \sin \left(2^{n} \pi \theta\right) d \theta
$$

represents a harmonic and bounded function with values on $\mathbb{R}$. By Fatou's theorem, for each $n=0,1,2, \ldots$, there exists a measurable set $A_{n} \subset[0,1]$ with Lebesgue measure equal to 0 such that

$$
\lim _{r \rightarrow 1} \int_{0}^{1} P_{r}(t-\theta) \sin \left(2^{n} \pi \theta\right) d \theta=\sin \left(2^{n} \pi t\right)
$$

for every $t \in[0,1] \backslash A_{n}$.

Let $A=\bigcup_{n=0}^{\infty} A_{n}$, thus $A$ has Lebesgue measure equal to 0 , and for each $t \in[0,1] \backslash A$, the limit (11) exists for every $n=0,1,2, \ldots$ 
Take $t \in[0,1] \backslash A$ and let it be fixed. If there were an element $f(t) \in c_{0}$, let us say $f(t)=\left(f_{n}(t)\right)_{n=0}^{\infty}$ such that

$$
\lim _{r \rightarrow 1} \varphi\left(r e^{2 \pi i t}\right)=f(t)
$$

with respect to the norm $\|\cdot\|_{\infty}$, then using the fact that the convergence with respect to $\|\cdot\|_{\infty}$ implies usual convergence on each coordinate, we would have

$$
f_{n}(t)=\sin \left(2^{n} \pi t\right)
$$

for each $n=0,1,2, \ldots$ However, the sequence $\left(\sin \left(2^{n} \pi t\right)\right)_{n=0}^{\infty} \notin c_{0}$ unless $t \in D$ where

$$
D=\left\{k / 2^{m}: k \in \mathbb{Z} \text { and } m \in \mathbb{N} \cup\{0\}\right\}
$$

which is a denumerable set and hence, $D \cap[0,1]$ has Lebesgue measure equal to 0 .

This shows that for at most a denumerable subset of $[0,1] \backslash A$, the limit (2) might exist, or, in other words, there is a Lebesgue subset $E \subset[0,1]$ with positive measure, where $\varphi$ does not have radial limits.

Before concluding, it should be mentioned what is really happening in the previous example: the Banach space under consideration $\left(c_{0},\|\cdot\|_{\infty}\right)$ does not satisfy the Radon-Nikodym property; roughly speaking, it states that the classical Radon-Nikodym theorem holds in the space. The Radon-Nikodym property has been extensively studied (for a detailed exposition see 4]) and has many formulations, among them that one stated in the following result proved by several authors, for example [2]

TheOREM 2 ([2]). A Banach space $X$ has the Radon-Nikodym property if and only if for every harmonic and bounded function $u: D \rightarrow X$ there exists an essentially bounded function $f:[0,1] \rightarrow X$ such that

$$
\lim _{r \rightarrow 1} u\left(r e^{2 \pi i t}\right)=f(t)
$$

for almost every $t \in[0,1]$.

In view of Theorem 2 , the space $\left(c_{0},\|\cdot\|_{\infty}\right)$ does not have the Radon-Nikodym property.

\section{REFERENCES}

[1] ASH, J. M.-KARAEV, M. T.: On the boundary behavior of special classes of $C^{\infty}$ functions and analytic functions, Internat. Math. Forum 7 (2012), 153-166.

[2] BLASCO, O.: Radon-Nikodym versus Fatou, in: 29th National Congress of the Mexican Mathematical Society (F. Avila Murillo et al., eds.), San Luis Potosí, México, 1996. Sociedad Matemática Mexicana. Aportaciones Mat., Comun., Vol. 20, México City, 1997, pp. $71-76$. 


\section{ON THE EXISTENCE OF RADIAL LIMITS}

[3] CONWAY, J. B.: Functions of One Complex Variable II, in: Grad. Texts in Math., Vol. 159, Springer-Verlag, New York, 1995.

[4] DIESTEL, J.-UHL, J. J.: Vector Measures, in: Math. Surveys Monogr., Vol. 15, AMS, Providence, R.I., 1977.

[5] FATOU, P.: Séries trigonométriques et séries de Taylor, Acta Math. 30 (1906), 335-400.

[6] SILVERMAN, R. A.: Complex Analysis with Applications. Prentice Hall, Englewood Cliffs, N. J., 1974.

[7] STEIN, E. M.-SHAKARCHI, R.: Real Analysis. Measure Theory, Integration, and Hilbert Spaces, in: Princeton Lect. Anal., Vol. 3, Princeton University Press, Princeton, NJ, 2005.

[8] ZYGMUND, A.: Trigonometric Series (3rd ed.), in: Cambridge Math. Lib. (With a foreword by R. Fefferman), Cambridge University Press, Cambridge, 2002.

Received July 24, 2013

Departamento de Matemáticas

Universidad de Sonora

Rosales y Luis Encinas

Hermosillo, Sonora, 83000

MÉXICO

E-mail:martha@gauss.mat.uson.mx crobles@gauss.mat.uson.mx 